\title{
The Effect of Electromagnetic Field Generated by a Mobile Phone on the Performance of a SPECT Scanner
}

\author{
A Quantitative Study
}

\author{
Ali Mahmoud Pashazadeh, MSc, Forough Jafarian Dehkordi, MSc, Kaveh Tanha, MSC, and Majid Assadi, MD
}

\begin{abstract}
Purpose: The aim of the current attempt was quantitative investigation of the electromagnetic interference (EMI) of a mobile phone with the function of a SPECT gamma camera during data acquisition.

Materials and Methods: We tested the effect of a mobile phone, in both ringing mode and standby mode, on one SPECT gamma camera during scanning a cylindrical phantom containing $5.4 \mathrm{mCi}{ }^{99 \mathrm{~m}} \mathrm{Tc}$. The experiment was performed for different distances of $10,12,14,16,18,20,25$, and $30 \mathrm{~cm}$ between mobile phone and head of the scanner, and for different head angles of 0,30, 60, 90, 120, 150, and 180 degrees. A RF-EMF meter measured strength of electromagnetic field throughout the study. Statistically significant decrease in count number was considered to be electromagnetic interference.

Results: There was significant reduction in the recorded counts during ringing of the mobile phone in all studied distances. For gamma camera, fixed at a distance, there was no uniform pattern of reduction of the counts at different angles between two operation modes of the mobile phone.

Conclusions: A mobile phone, at close distance, can be a sensible source of electromagnetic field, disturbing the normal function of a gamma camera.
\end{abstract}

Key Words: quantitative, electromagnetic interference, mobile phone, SPECT, gamma camera

(Clin Nucl Med 2015;40: 545-547)

$\mathrm{E}$ lectromagnetic interference (EMI) of mobile phones with the function of medical devices is an unfavorable event that may result in malfunctioning of this life-supporting equipment. ${ }^{1-6}$ Today, mobile phones have been translated from a just communication tool into can-not-live-without device, increasing the probability that it may come to the vulnerable medical devices.

Although, up to now, number of reported significant medical errors that resulted from mobile phone interference with medical devices have been relatively rare, yet in the literature several cases of EMI and concomitant errors have been published. ${ }^{7-9}$ Results of the studies have indicated that based on the factors such as type of medical device and mobile phone, and also separation distance between these two, different levels of EMI may occur. ${ }^{10}$ Gamma camera of a SPECT scanner is part of medical systems that may be susceptible to the electromagnetic field (EMF) generated by mobile phone. ${ }^{7,11,12}$ SPECT is a routine imaging modality that provides images from bio-distribution of radioisotopes inside the body. The first clinical evidence of mobile phone EMI with SPECT scanner was a case report in which function of a gamma camera had been interrupted by ringing of a mobile phone, located in a patient's pocket, as light spots on frame 6 of the flow phase in the acquired image during the renal scintigraphy. ${ }^{7}$ This vulnerability is largely because of the photomultiplier tubes (PMTs) that are used to intensify acquired

Received for publication August 25, 2014; revision accepted February 12, 2015. From the Persian Gulf Nuclear Medicine Research Center, Bushehr University of Medical Sciences, Bushehr, Iran.

This study was financially supported by Bushehr University of Medical Sciences. Conflicts of interest and sources of funding: none declared.

Reprints: Ali Mahmoud Pashazadeh, MSc, The Persian Gulf Nuclear Medicine Research Center, The Persian Gulf Biomedical Sciences Institute, Boostan 19 Alley,

Sangi St, Bushehr, Iran. E-mail: a.pashazadeh@bpums.ac.ir.

Copyright (C) 2015 Wolters Kluwer Health, Inc. All rights reserved.

ISSN: 0363-9762/15/4007-0545 signal of the detected photons. ${ }^{13}$ Electromagnetic interference with the function of photomultiplier tubes, and consequently with the operation of gamma camera, dates back before the 1980s in which former models of SPECT scanners were being affected by the earth's magnetic field during rotation of head gantry. ${ }^{14}$ Whereas from those observations onwards, magnetic shielding has been applied in PMT designing to provide protection and stability necessary for acceptable function of gamma cameras in the presence of the earth's magnetic field, yet this shielding seems not to be well enough to avoid interference by mobile phone's generated field. In the former study, we qualitatively investigated such interference based on visual interpretation of a nuclear medicine physician. ${ }^{11}$ Results of that evaluation, performed by 7 different models of mobile phones, indicated that some types of mobile phones have the capability to induce interruption on the function of the gamma camera leading to a decrease in the recorded counts of the ${ }^{99 \mathrm{~m}} \mathrm{Tc}$ activity. ${ }^{11}$ As our previous attempt ${ }^{1}$ was a qualitative rather than a quantitative study, which has its limitations, therefore in this study we were going to study EMI of a mobile phone on the function of a gamma camera in a quantitative way.

\section{METHODS}

\section{Technical Equipment}

The study was performed on a rotating dual-head digital gamma camera (ADAC Pegasys; model SH Genesys Epic, Milpitas, CA), located in our nuclear medicine clinic, that was equipped with a low-energy allpurpose parallel whole collimator.

To generate an electromagnetic field, one Nokia 3350 (Nokia, Finland) mobile phone, operated with GSM communication system in the frequency of $900 \mathrm{MHz}$, was chosen for the study and placed in the center of rotation of the scanner.

Measurements of the EMF strength throughout the study were done using a RF-EMF meter (EXTECH, USA).

Source of activity consisted of $5.4 \mathrm{mCi}{ }^{99} \mathrm{~m} \mathrm{Tc}$ that was diluted in about $17 \mathrm{~cm}^{2}$ of water. Then the solution was poured inside a cylindrical phantom with the dimension of 27 (diameter) $\mathrm{mm} \times 142$ (length) $\mathrm{mm}$ and was placed near the mobile phone.

To assess the effects of distance on induced EMI, we used one of the camera's head. Head of the camera was fixed at a 90-degree angle, then the study was performed at 8 different distances of $10,12,14,16$, $18,20,25$, and $30 \mathrm{~cm}$.

Also, to evaluate the effect of head angle, SPECT camera was set at the radius of rotation (ROR) of $30 \mathrm{~cm}$, then phantom was scanned at different angles of $0,30,60,90,120,150$, and 180 degrees by both heads. For each angle, recorded counts of 2 heads were averaged to calculate the average of the counts of that particular angle.

In each scenario, the function of the gamma camera was tested in standby and ringing modes of mobile phone, and for each mode, activity was scanned for $10 \mathrm{~s}$. The criterion that was considered as interference of the mobile phone with the function of the camera was significant reduction in the recorded counts of radioactivity in each scan.

To do statistical evaluation of the data, all steps of the study were repeated three times and SD of the measurements was calculated. 
TABLE 1. Recorded Counts of ${ }^{99 \mathrm{~m}} \mathrm{Tc}$ by Gamma Camera During $10 \mathrm{~s}$ in Standby and Ringing Modes of the Mobile Phone at Different Angles of Camera Head

\begin{tabular}{|c|c|c|c|c|c|c|c|c|}
\hline \multicolumn{2}{|c|}{ Angle of the Camera (Degrees) } & \multirow{2}{*}{$\begin{array}{c}\mathbf{0} \\
67,500 \pm 228\end{array}$} & \multirow{2}{*}{$\begin{array}{c}\mathbf{3 0} \\
69,701 \pm 633\end{array}$} & \multirow{2}{*}{$\begin{array}{c}\mathbf{6 0} \\
64,837 \pm 222\end{array}$} & \multirow{2}{*}{$\begin{array}{c}90 \\
63,064 \pm 666\end{array}$} & \multirow{2}{*}{$\begin{array}{c}120 \\
65,487 \pm 117\end{array}$} & \multirow{2}{*}{$\begin{array}{c}150 \\
67,825 \pm 134\end{array}$} & \multirow{2}{*}{$\frac{180}{67,465 \pm 231}$} \\
\hline ecorded counts $( \pm \mathrm{SD})$ & Standby mode & & & & & & & \\
\hline & Ringing mode & $65,325 \pm 702$ & $68,305 \pm 208$ & $62,951 \pm 38$ & $62,765 \pm 152$ & $63,256 \pm 123$ & $67,456 \pm 200$ & $64,987 \pm 787$ \\
\hline
\end{tabular}

TABLE 2. Recorded Counts of ${ }^{99 \mathrm{~m}}$ Tc by Gamma Camera During $10 \mathrm{~s}$ in Standby and Ringing Modes of the Mobile Phone at Different Distances From Camera Head

\begin{tabular}{|c|c|c|c|c|c|c|c|c|c|}
\hline $\begin{array}{l}\text { Distance of the Came } \\
\text { Phantom/Mobile Phol }\end{array}$ & $\begin{array}{l}\text { Head From } \\
\text { e }(\mathrm{cm})\end{array}$ & 10 & 12 & 14 & 16 & 18 & 20 & 25 & 30 \\
\hline \multirow[t]{2}{*}{ Recorded count $( \pm \mathrm{SD})$} & Standby mode & $78,892 \pm 250$ & $77,402 \pm 82$ & $76,408 \pm 98$ & $75,670 \pm 226$ & $74,848 \pm 184$ & $74,323 \pm 123$ & $73,751 \pm 335$ & $73,165 \pm 53$ \\
\hline & Ringing mode & $77,922 \pm 185$ & $76,879 \pm 67$ & $76,115 \pm 27$ & $75,452 \pm 180$ & $74,286 \pm 226$ & $73,747 \pm 158$ & $73,040 \pm 125$ & $72,550 \pm 84$ \\
\hline
\end{tabular}

Comparison of the data was done with statistical test of two-way repeated measures.

\section{RESULTS}

In this study, to quantitatively assess mobile phone EMI with the function of a SPECT gamma camera, the operation of the scanner was tested at different RORs and different rotation angles when one mobile phone was being placed at the center of the rotation. Results of the study including rotation angles, RORs, and corresponding recorded counts in standby and ringing modes in each scenario are summarized in Tables 1 and 2.

Measurements of the electromagnetic field of mobile phone with RF-EMF meter revealed that this quantity decreased exponentially as the distance from mobile phone increased (Fig. 1). A similar falling trend was observed in recorded counts of ${ }^{99 \mathrm{~m}} \mathrm{Tc}$ activity by gamma camera for both standby and ringing modes of mobile phone, when distance of gamma camera from center of rotation increased. Also for each distance, recorded counts of activity were significantly lower when mobile phone was operating in ringing mode than in standby mode (Fig. 2)

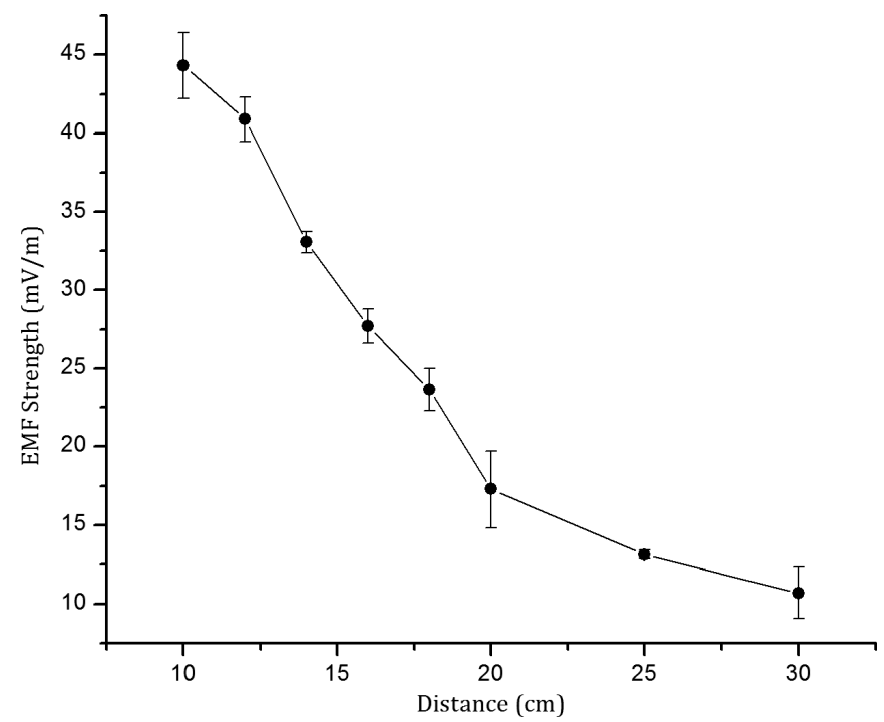

FIGURE 1. Electromagnetic field of mobile phone at different distances when operated in ringing mode.
$(P<0.05)$. The only exception for this observation was at a distance of $16 \mathrm{~cm}$ in which the difference was insignificant.

Analysis of the data of the rotation test revealed that recorded count by gamma camera, fixed at ROR of $30 \mathrm{~cm}$, has different values at different angles, and in some angles this difference is statistically significant (Fig. 3).

\section{DISCUSSION}

The aim of the present attempt was quantitative investigation of electromagnetic interference of mobile phone with the function of a SPECT scanner. In fact, we considered a hypothetical situation in which mobile phone may come in close distance of a gamma camera. Interruption in normal operation of a gamma camera, as visual reduction in the recorded counts of the ${ }^{99 \mathrm{~m}} \mathrm{Tc}$ that resulted from mobile phone field, in our previous study encouraged us to set an experiment to quantitatively study the phenomenon. The criterion for interference was statistically significant difference in the counts of the ${ }^{99 \mathrm{~m}} \mathrm{Tc}$ recorded by the scanner.

Based on the RF-EMF meter measurements, electromagnetic field generated by mobile phone, when it was in standby mode, was

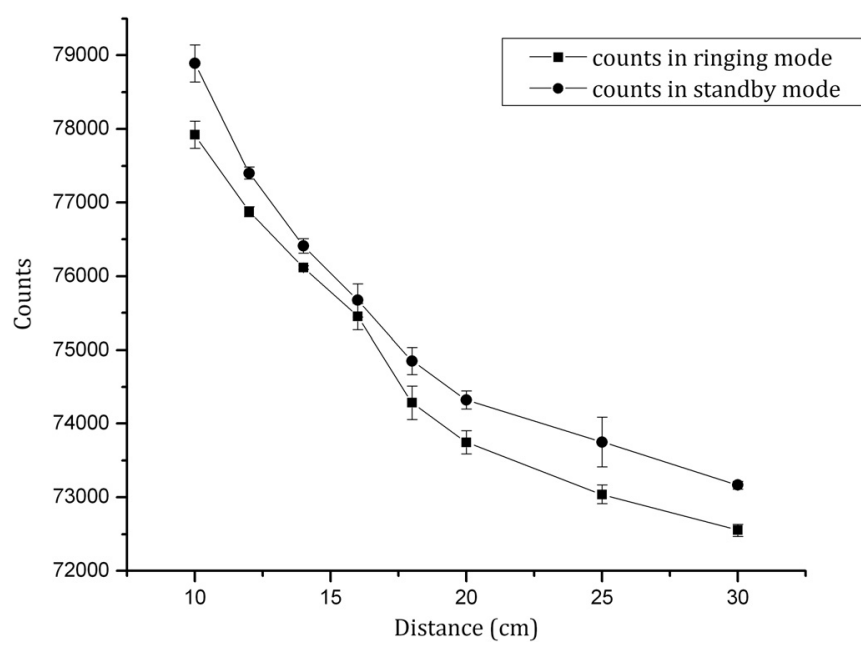

FIGURE 2. Recorded counts of ${ }^{99 m}$ Tc activity by gamma camera at different distances from center of rotation when mobile phone is operated in standby and ringing modes. 


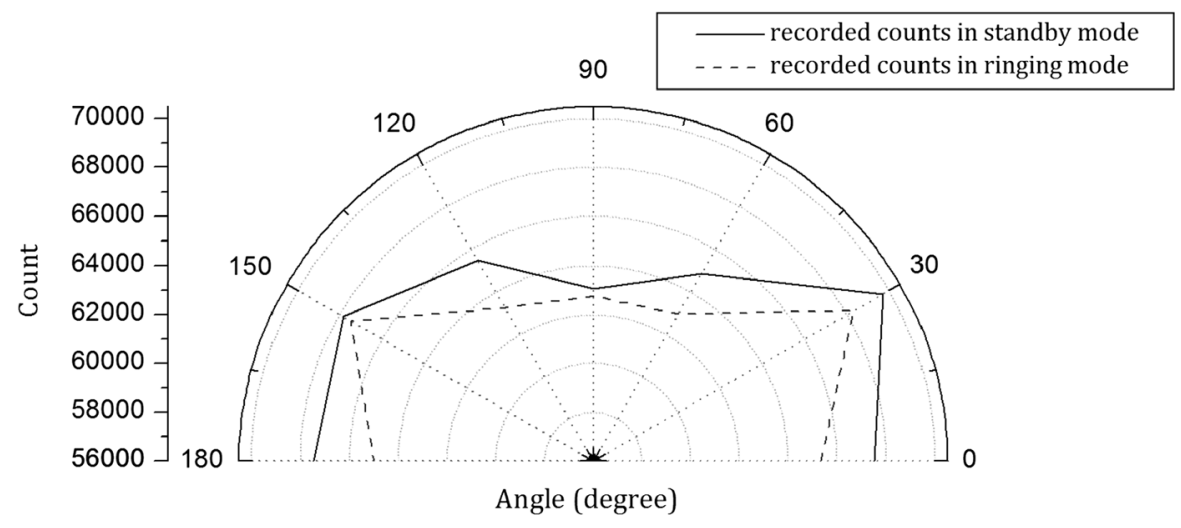

FIGURE 3. Recorded counts of ${ }^{99 \mathrm{~m}} \mathrm{Tc}$ activity by gamma camera at different rotation angles when mobile phone is operated in standby and ringing modes.

of the order of background level, and there was no significant change in this quantity before and after the presence of mobile phone in its position in the center of the SPECT scanner in this mode. But, whenever its operation status changed into ringing mode, RF-EMF meter showed remarkable increase in the electromagnetic field, indicating potential of mobile phone to remarkably enhance EMF of the environment in its vicinity.

We observed that at close distance, less than $30 \mathrm{~cm}$, ringing of mobile phone is able to adversely affect function of a gamma camera, although there was an exception in distance of $16 \mathrm{~cm}$. Therefore, we can conclude that ringing of mobile phone carried by the patient under scan may have unfavorable effect on acquired data during gamma scan. This can be more highlighted in cases when the head of the camera is in close contact with the patient, a situation that may take place during renal scintigraphy.

According to measurements, strength of EMF generated by mobile phone at a distance of $10 \mathrm{~cm}$ was four times more than that $30 \mathrm{~cm}$. This observation highlights the importance of distance on the strength of EMF field. Perhaps, one-meter policy, recommended by some researches, provides a fairly secure zone to avoid such interference. In a similar study by Stegmayr et al to assess potential of mobile phone and digitally enhanced cordless telecommunication (DECT) device to induce adverse impact on SPECT, they found that while at distances of 0,30 , and $42 \mathrm{~cm}$, interference may occur, but at greater than $2 \mathrm{~m}$, any effect was not observed. ${ }^{12}$

After SPECT was set at ROR of $30 \mathrm{~cm}$ and operated at step and shoot mode with interval angles of 30 degrees, we observed that while for some angles the differences in the recorded counts were significant, for other angles such change was not observed. On the other hand, the pattern of malfunctioning of device in both standby and dialing modes of the mobile phone was non-uniform. The reason behind such observation perhaps lay in the spatial distribution of the electromagnetic field. Situations such as variable noise in the room, room geometry, materials used in the room walls, and reflexing factors in the environment of the study may affect distribution pattern and hence strength of the field, the situations that were not under our control to avoid.

Based on the results of the present study, we conclude that there is a certain level of EMF as background that SPECT is well protected against such amount of EMF. But mobile phone as a portable source of electromagnetic field may change this level when ringing. It was the reason that the presence of mobile phone operating in standby mode near the SPECT gamma camera had no sensible effect on the function of the camera, but in ringing mode it leads to malfunctioning of the scanner as a significant decrease in the recorded counts by the camera.

\section{ACKNOWLEDGMENTS}

The authors would like to thank Dr. Mohammad Seyedabadi for his kind help during statistical analysis of the data. We also thank Mrs Narges Jamili who helped us to collect data.

\section{REFERENCES}

1. Mahmoud Pashazadeh A, Aghajani M, Nabipour I, et al. An update on mobile phones interference with medical devices. Radiat Prot Dosimetry. 2013;156: 401-406.

2. Ang G, Lian P, Ng W, et al. Digital mobile telephones and interference of ophthalmic equipment. Eye. 2005;21:29-32.

3. Dang BP, Nel PR, Gjevre JA. Mobile communication devices causing interference in invasive and noninvasive ventilators. J Crit Care. 2007;22:137-141.

4. Fernández-Chimeno M, Silva F, eds. Mobile phones electromagnetic interference in medical environments: a review. IEEE International Symposiumon Electromagnetic Compatibility (EMC). 2010:311-316.

5. Tandogan I, Temizhan A, Yetkin E, et al. The effects of mobile phones on pacemaker function. Int J Cardiol. 2005;103:51-58.

6. Hekmat K, Salemink B, Lauterbach G, et al. Interference by cellular phones with permanent implanted pacemakers: an update. Europace. 2004;6:363-369.

7. Azizmohammadi Z, Baharfar N, Javadi H, et al. Effect of mobile cell phone ringing on function of gamma camera. Clin Nucl Med. 2011;36:1131.

8. Tri JL, Hayes DL, Smith TT, et al., eds. Cellular phone interference with external cardiopulmonary monitoring devices. Mayo Clinic Proceedings; 2001: Elsevier.

9. Van Lieshout EJ, Van Der Veer SN, Hensbroek R, et al. Interference by newgeneration mobile phones on critical care medical equipment. Crit Care. 2007; 11:165.

10. Cohen T, Ellis WS, Morrissey JJ, et al. Safe use of cellular telephones in hospitals: fundamental principles and case studies. J Healthc Inf Manag. 2005;19:39.

11. Javadi H, Azizmohammadi Z, Mahmoud Pashazadeh A, et al. The electromagnetic interference of mobile phones on the function of a gamma-camera. Clin Nucl Med. 2014;39:232-236.

12. Stegmayr A, Fessl B, Hortnagl R, et al. Interference of mobile phones and digitally enhanced cordless telecommunications mobile phones in renal scintigraphy. Clin Nucl Med. 2013;38:597-603.

13. Leonora E, Aiello S, Leotta G, eds. Influence of the Earth's magnetic field on large area photomultipliers. Advancements in Nuclear Instrumentation Measurement Methods and their Applications (ANIMMA). IEEE 2nd International Conference. 2011;1259-1267.

14. Rogers WL, Clinthorne NH, Harkness BA, et al. Field-flood requirements for emission computed tomography with an Anger camera. J Nucl Med. 1982;23: $162-168$. 